\title{
Shugoshin protects centromere pairing and promotes segregation of nonexchange partner chromosomes in meiosis
}

\author{
Luciana Previato de Almeida a, Jared M. Evatt ${ }^{\mathrm{a}, \mathrm{b}}$, Hoa H. Chuonga, Emily L. Kurdzo ${ }^{\mathrm{a}, \mathrm{b}}$, Craig A. Eyster ${ }^{\mathrm{a}}$, \\ Mara N. Gladstone ${ }^{\mathrm{a}, c}$, Laura Gómez- ${ }^{\mathrm{d}}$, Elena Llano ${ }^{\mathrm{d}}$, Régis Meyer ${ }^{\mathrm{a}}$, Alberto M. Pendas ${ }^{\mathrm{d}}$, Roberto J. Pezza ${ }^{\mathrm{a}, \mathrm{b}, 1}$, \\ and Dean S. Dawson ${ }^{a, b, 1}$
}

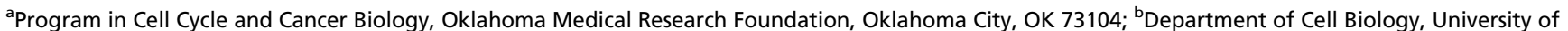
Oklahoma Health Science Center, Oklahoma City, OK 73104; ' Program in Molecular Microbiology, Sackler School of Biomedical Science, Tufts University, Boston, MA 02111; and d Molecular Mechanisms Program, Centro de Investigación del Cáncer, Instituto de Biología Molecular y Celular del Cáncer, Centro Superior de Investigaciones Científicas-Universidad de Salamanca, 37007 Salamanca, Spain
\end{abstract}

Edited by Terry L. Orr-Weaver, Whitehead Institute, Cambridge, MA, and approved April 1, 2019 (received for review February 12, 2019)

Faithful chromosome segregation during meiosis I depends upon the formation of connections between homologous chromosomes. Crossovers between homologs connect the partners, allowing them to attach to the meiotic spindle as a unit, such that they migrate away from one another at anaphase I. Homologous partners also become connected by pairing of their centromeres in meiotic prophase. This centromere pairing can promote proper segregation at anaphase I of partners that have failed to become joined by a crossover. Centromere pairing is mediated by synaptonemal complex (SC) proteins that persist at the centromere when the SC disassembles. Here, using mouse spermatocyte and yeast model systems, we tested the role of shugoshin in promoting meiotic centromere pairing by protecting centromeric synaptonemal components from disassembly. The results show that shugoshin protects the centromeric SC in meiotic prophase and, in anaphase, promotes the proper segregation of partner chromosomes that are not linked by a crossover.

shugoshin | meiosis | nonexchange chromosome | centromere pairing | PP2A

Eaitho aithful chromosome segregation during meiosis depends upon the formation of connections between homologous chromosome pairs. Crossovers, also called exchanges, are the basis of these connections. Chiasmata, the cytological manifestation of crossovers, and sister chromatid cohesion distal to the crossover create a physical link that holds homologous chromosomes in pairs called bivalents (reviewed in ref. 1). The linkage allows the bivalent to attach to the meiotic spindle as a single unit, such that at anaphase I, the partners will migrate away from one another to opposite poles of the spindle. However, sometimes, even in the absence of exchanges, proper meiotic chromosome segregation is achieved (reviewed in ref. 2). In yeast and Drosophila when a single chromosome pair does not experience an exchange, it still usually segregates correctly (3-6). Although nonexchange chromosomes have been difficult to study in mammalian models, there may be mechanisms beyond crossing-over that help to direct their behavior in meiosis I. For example, in mice, the majority of chromosomes in oocytes from Mlh1 recombination-deficient mutants appeared to be spatially balanced on the spindle (7), and in humans, while smaller chromosomes fail to experience crossovers in about $5 \%$ of meioses (8-10), they are estimated to nondisjoin in less than $1 \%$ of meioses (9-11).

In yeast and Drosophila, the centromeres of nonexchange partners pair or interact in meiotic prophase (12-15). Similar centromere pairing is also seen in mouse spermatocytes $(16,17)$. Meiotic centromere pairing (or clustering in Drosophila females) is mediated by proteins that are components of the synaptonemal complex (SC) (14-18). In Drosophila, centromere proteins (CENP-C, CAL1) and the meiotic cohesion protein ORD have also been shown to be required for centromere clustering (18-21).
The SC zippers the axes of homologous chromosomes along their lengths in midmeiotic prophase (pachytene) and disassembles in late prophase (diplotene). However, all SC components tested [Zip1 in yeast, its functional homologs SYCP1 in mice, and C(3)G in Drosophila; the mouse SC components SYCE1, SYCE2, SYCE3, and TEX12; and the Drosophila protein Cona] persist at centromeres, holding them together in pairs (14-18). In budding yeast, this centromere pairing is correlated with proper disjunction of the nonexchange pair $(14,15)$.

Important questions regarding the mechanism and function of centromere pairing remain unanswered. First, how does centromere pairing in prophase ensure disjunction in anaphase? This is curious, as in budding yeast and mice, the SC components that are protected at the centromeres in late prophase (Zip1/ SCYP1) are greatly reduced or undetectable when the centromeres begin attaching to the microtubules of the spindle $(14,16$, 17). Second, what enables SC proteins to persist at the paired centromeres when the SC disassembles?

The persistence of centromeric SC in late prophase is reminiscent of the protection of meiotic cohesins at centromeres at the metaphase I to anaphase I transition when arm cohesion is

\section{Significance}

Meiotic crossovers form a connection between homologous chromosomes that allows them to attach to the spindle as a single unit in meiosis I. In humans, failures in this process are a leading cause of aneuploidy. A recently described process, called centromere pairing, can also help connect meiotic chromosome partners in meiosis. Homologous chromosomes become tightly joined by a structure called the synaptonemal complex (SC) in meiotic prophase. After the SC disassembles, persisting SC proteins at the centromeres mediate their pairing. Here, studies in mouse spermatocytes and yeast are used to show that the shugoshin protein helps SC components persist at centromeres and helps centromere pairing promote the proper segregation of yeast chromosomes that fail to become tethered by crossovers.

Author contributions: E.L.K., R.M., A.M.P., R.J.P., and D.S.D. designed research; L.P.d.A., J.M.E., H.H.C., E.L.K., C.A.E., M.N.G., L.G.-H., E.L., R.M., A.M.P., R.J.P., and D.S.D. performed research; and D.S.D. wrote the paper.

The authors declare no conflict of interest.

This article is a PNAS Direct Submission.

This open access article is distributed under Creative Commons Attribution-NonCommercialNoDerivatives License 4.0 (CC BY-NC-ND).

${ }^{1}$ To whom correspondence may be addressed. Email: Roberto-Pezza@omrf.org or dawsond@omrf.org.

This article contains supporting information online at www.pnas.org/lookup/suppl/doi:10. 1073/pnas.1902526116/-/DCSupplemental.

Published online April 24, 2019. 
lost (reviewed in ref. 22). Protection of centromeric cohesin in meiosis I is mediated by shugoshin - a function first revealed by studies of the gene mei-S322 in Drosophila $(23,24)$. In yeasts and mouse oocytes, shugoshin recruits PP2A phosphatase to centromeres, rendering the centromeric cohesin refractory to cleavage by the protease separase at the metaphase I to anaphase I transition (25-29). Shugoshins also interact with other proteins (Mad2, MCAK, the chromosome passenger complex, and cohesin subunits) to modulate chromosome structure and segregation in meiosis and mitosis (27, 30-34). In budding and fission yeast, shugoshin acts by protecting the Rec8 component of cohesin from phosphorylation by casein kinase and also in budding yeast by the Dbf4 kinase (DDK). In other organisms the identities of the kinases that prepare cohesins for separase cleavage have not been determined (35-37).

Phosphorylation also promotes SC disassembly and degradation, but at the pachytene-to-diplotene transition (reviewed in ref. 38). Studies in rats and mice have correlated the phosphorylation of SC components (SYCP1, SYCP3, TEX12, and SYCE1) with pachytene exit and SC disassembly $(39,40)$ and the Polo-like kinase PLK1 localizes to the SC central element in pachytene and can phosphorylate SYCP1 and TEX12 in vitro (39). Similarly, in budding yeast, Polo-like kinase (Cdc5) expression is central in promoting SC disassembly (41), but it works in a network with other kinases, namely Dbf-4 kinase and cyclindependent kinase (CDK) (39, 42-45).

The parallels between the protection of cohesins and SC components at the centromeres compelled us to explore whether shugoshin is responsible for protecting centromeric SC from disassembly signals upon pachytene exit. Our cytological experiments with mouse spermatocytes revealed that this is the case, while genetic approaches with budding yeast revealed that shugoshin is necessary for mediating the segregation of nonexchange chromosome pairs that depend upon centromere pairing for their meiotic segregation fidelity.

\section{Results and Discussion}

Centromere Pairing Links Homolog Pairs in the Absence of Chiasmata. In chromosome spreads from crossover competent mice about 4-5\% of prophase chromosomes appear to be achiasmate (nonexchange) (46-49). Previous studies have shown that homologous partners that appear to be achiasmate are aligned along their arms and are connected at their centromeres by a short block of persisting SC $(16,17)$. These observations suggest that mechanisms exist to form connections between homologous centromeres without the need for homologous recombination. An alternative explanation is that these apparently nonexchange chromosomes are actually connected by crossovers that do not yield obvious chiasmata in chromosome spreads. If centromere pairing is dependent upon the formation of crossovers that cannot be visualized in chromosome spreads, then in mutants unable to form crossovers, centromere pairing should be greatly reduced. To test this, we examined centromere pairing in mice with mutations that compromise the two major crossover formation pathways. In mice, $H f m 1$ is essential to the formation of class I crossovers. This pathway gives rise to $\sim 90 \%$ of crossovers (class I crossovers) $(50,51)$. Mus81 is essential for the efficient formation of rarer class II crossovers (52). We immunostained spermatocyte chromosome spreads for SYCP3 and SYCP1, components of the lateral and transverse filaments of the SC, in spermatocytes from wild-type, $H \mathrm{fm} 1^{-/-}$, and $H \mathrm{fm} 1^{-/-} / \mathrm{Mus}_{\mathrm{S}} \mathrm{1}^{-/-}$mice. SYCP3 immunostaining enabled visualization of chromosome cores and SYCP1 was used to monitor the persistence of SC components at paired centromeres after SC disassembly (centromere pairing) $(16,17)$. In each chromosome spread we counted the number of chromosome pairs that exhibited no clear chiasmata, whether or not their centromeres were paired (Fig. $1 A$ and $B$ ). As expected, elimination of the class I or class I and class II pathways
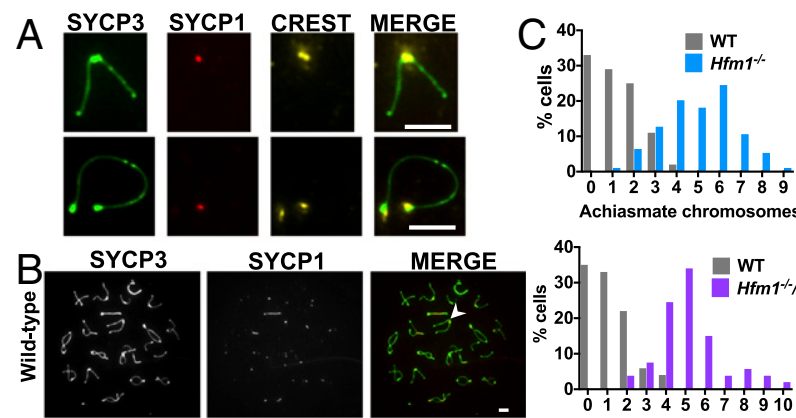

SYCP1
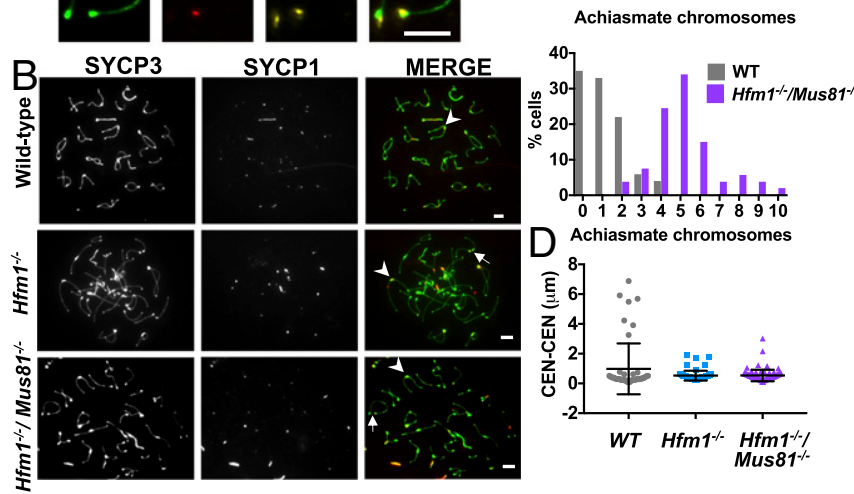

Fig. 1. Centromeres pair efficiently in the absence of chiasmata. (A) Examples of apparently achiasmate partners exhibiting pairing (Top) or no pairing (Bottom) of their centromeres. Indirect immunofluorescence was used to detect SYCP3 (chromosome axes, green), SYCP1 (synaptonemal complex, red), or centromeres (CREST antibody, yellow) in chromosome spreads from mouse spermatocytes. The centromeric end of mouse chromosomes features a bulbous focus of SYCP3 staining and SYCP1 persists at the centromere after SC disassembly $(16,17)$. (Scale bars, $5 \mu \mathrm{m}$.) (B) Representative middiplotene chromosome spreads from wild-type, $\mathrm{Hfm}^{-/-}$, and $\mathrm{Hfm}^{-1-} \mathrm{Mus} 81^{-1-}$ mice. Arrowheads indicate apparently achiasmate chromosomes with paired centromeres. Arrows indicate apparently achiasmate chromosome partners with unpaired centromeres. $(C)$ Achiasmate chromosome frequency in spermatocytes from wild-type and recombination-deficient mice. Chromosome spreads were scored for the number of clearly achiasmate chromosomes per cell (some chromosomes could not be clearly resolved, and thus the graphs underestimate the achiasmate frequency). Chromosome spreads were from wildtype and mutant littermates. (C, Top) WT (gray, $n=100$ cells) and $\mathrm{Hfm}^{-1-}$ mutants (blue, $n=94$ cells). (C, Bottom) WT (gray, $n=51$ cells) and $\mathrm{Hfm}^{-1}$ Mus $81^{-1-}$ mutants (purple, $n=53$ cells). $(D)$ The distance between the centromeres was measured for apparently achiasmate partners in the chromosome spreads used for the experiment in C. WT, $n=51$ chromosomes; $\mathrm{Hfm}^{-/-}$, $n=69$ chromosomes; $\mathrm{Hfm} 1^{-/-}$Mus $81^{-1-}, n=87$ chromosomes.

resulted in many more achiasmate chromosomes per cell (Fig. $1 C$ ) $(50,51)$. If centromere pairing of apparently achiasmate chromosomes in wild-type mice $(16,17)$ depends upon undetectable crossovers, then in the recombination mutants achiasmate chromosomes should have a reduced frequency of centromere pairing. To test this, we measured the distance between the centromeres of achiasmate chromosome partners in $\mathrm{WT}, \mathrm{Hfml}^{-/-}$, and $\mathrm{Hfml}^{-/-}$ Mus $81^{-/-}$mice (Fig. 1D). The loss of recombination did not diminish centromere pairing efficiency (Fig. $1 D$ and SI Appendix, Fig. $\mathrm{S} 1)$. This result suggests that paired centromeres of achiasmate partners are not held together by undetectable crossovers, but rather by persisting SC at the centromeres $(16,17)$.

Shugoshin 2 Protects Centromere Pairing in Mice. Shugoshin 2 (SGO2) localizes to the centromeres of chromosomes in mouse spermatocytes (53). We compared the centromeric localization of SGO2 with the timing of SC protection at centromeres (Fig. $2 A$ ). The axial/lateral element component SYCP3 was used as a marker for the SC. SGO2 is first detected at centromeres in early diplotene cells (Fig. $2 A$ and $B$ ) and is still there in mid- and late diplotene. This corresponds with the time at which SC components are removed from chromosome arms but not from centromeres $(16,17)$. Thus, these data are consistent with the model that SGO2 protects the centromeric SC from the disassembly process.

To test whether SGO2 is necessary for the protection of centromeric SC, we monitored the persistence of centromeric 

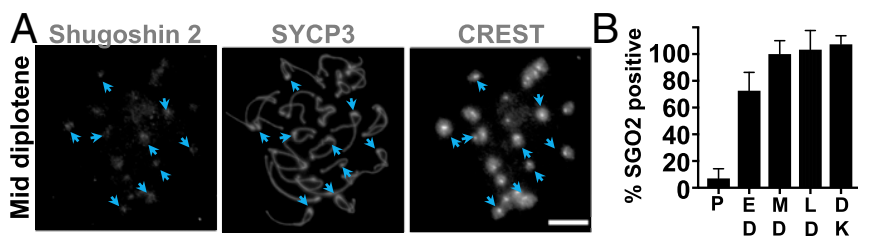

Fig. 2. SGO2 colocalizes with persisting synaptonemal complex components at centromeres in prophase I. $(A)$ Indirect immunofluorescence was used to evaluate localization of SGO2 on chromosomes at stages of diplotene. Staining with CREST antibody was used to identify centromere regions. Arrowheads indicate examples of paired centromeres. (Scale bar, $5 \mu \mathrm{m}$ for all images.) (B) The average percentage of centromeres per spread showing colocalization of SGO2. DK, diakinesis; ED, early diplotene; LD, late diplotene; MD, middiplotene; $\mathrm{P}$, pachytene. The stage of each cell was determined by the SC morphology (Materials and Methods). Error bars indicate SD. A minimum of 20 spreads were scored for each category.

SYCP1 in Sgo $2^{-/-}$spermatocytes and wild-type control cells (Fig. 3). In wild-type cells SYCP1 persistence at centromeres mirrors that of other SC components $(16,17)$. In pachytene cells the SC of the $\mathrm{SgO}^{-/-}$mutants was indistinguishable from the wild-type control (Fig. $3 A$ and $B$ ). In early diplotene, the SYCP1 signal was visible at nearly all paired centromeres in wild-type chromosome spreads and at nearly $75 \%$ of the centromeres in the Sgo $2^{-/-}$mutants (Fig. 3C). Other SC components, SYCE1, SYCE2, and SIX6OS1, behaved similarly to SYCP1 (SI Appendix, Fig. S2). In wild-type spreads the SYCP1 persisted at the centromeres through diplotene (Fig. $3 \mathrm{C}$ ). But in $\mathrm{SgO}^{-/-}$mutants the percentage of centromeres with detectable SYCP1 staining was reduced in late diplotene (Fig. $3 A-C$ ). Thus, the absence of SGO2 did not detectably affect SC components in pachytene but did allow SC components to be lost from centromeres more quickly than in wild-type spermatocytes.

The heightened loss of SYCP1 from centromeres would predict that $\mathrm{SgO}_{\mathrm{O}} \mathrm{C}^{-1}$ spermatocytes would also have a defect in homologous centromere pairing in diplotene. In early diplotene, nearly all centromeres are paired in wild-type spermatocytes and pairing levels go down as cells proceed through diplotene (Fig. 3 $A$ and $D$ ). In contrast, by early diplotene in $S g o 2^{-/-}$cells many of the centromere pairs have already disengaged and pair levels go down through diplotene (Fig. $3 B$ and $D$ ). In both the wild-type control and the Sgo2 ${ }^{-/-}$chromosome spreads, the unpaired centromeres have significantly less SYCP1 staining than do the paired centromeres (Fig. $3 E$ and SI Appendix, Fig. S3), supporting the notion that it is the protection of SYCP1 that allows centromere pairing to persist.

Together these results suggest that wild-type and $\mathrm{Sgo}^{-/-}$ spermatocytes have similar SC structures and complete centromere pairing in pachytene. Importantly, in early diplotene in $\mathrm{SgO}^{-/-}$mutants, the SC is present at most centromeres and they remain paired. Thus, SGO2 is not necessary for establishing centromere pairing. However, centromere pairing disappears more rapidly in Sgo $2^{-/-}$spermatocytes, suggesting that $\mathrm{SGO} 2$ is necessary to maintain centromeric SC components, and centromere pairing, in diplotene.

PP2A Promotes Centromere Pairing in Mouse Spermatocytes. SGO2 could be protecting centromeric SC through the recruitment of one of its effector proteins to the centromere (reviewed in ref. 54). Shugoshin recruits PP2A phosphatase to meiotic centromeres in germ cells, where the PP2A opposes the phosphorylation of centromeric cohesins $(25,26,55)$. To test whether this mechanism is being used to protect centromeric SC from disassembly in diplotene, we evaluated the persistence of centromeric $\mathrm{SC}$, and centromere pairing, in spermatocytes when phosphatase activity was inhibited (Fig. 4). In these experiments we evaluated diplotene-like chromosome spreads from cultured spermatocytes (56) treated with the phosphatase inhibitors cantharidin and okadaic acid, which at the concentrations used preferentially inhibit PP2A over other phosphatases $(57,58)$. Treatment with either inhibitor significantly reduced the retention of SYCP1 at the centromeres and resulted in a substantial loss of centromere pairing (Fig. $4 A-D$ ), consistent with the model that PP2A protects SYCP1 at centromeres, although it is formally possible that other targets of these compounds could be involved.

Recent studies in Drosophila have suggested that PP2A and shugoshin might each act to promote localization of the other to the centromeres (59), but in our experiments no reduction in SGO2 localization was seen at the centromeres following addition of the phosphatase inhibitors (Fig. $4 A$ and $D$ ). Although it is possible that both inhibitors are achieving their effects through some other target, the fact that both of these PP2A inhibitors reduce $\mathrm{SC}$ protection at centromeres is consistent with the model that PP2A, recruited by SGO2, opposes kinase activities that promote SC disassembly at centromeres upon pachytene exit.

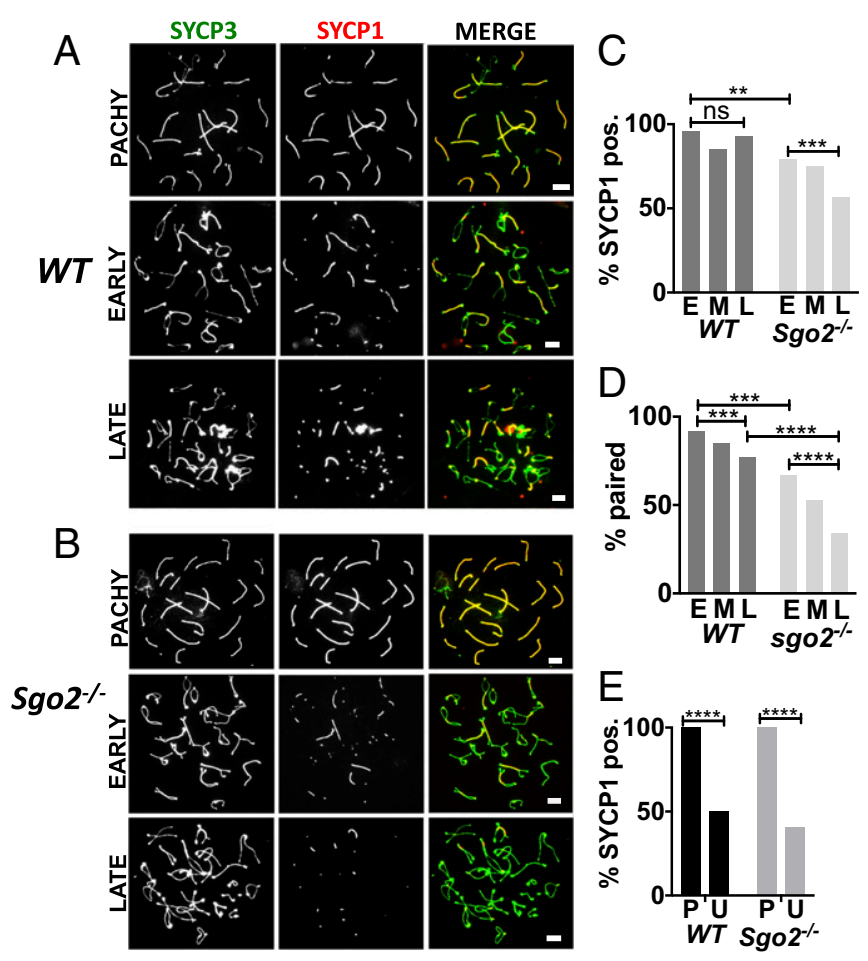

Fig. 3. $\mathrm{SGO} 2$ is required for the persistence of centromeric synaptonemal complex components in diplotene. Indirect immunofluorescence was used to monitor the morphology of chromosomes from wild-type and $\mathrm{Sgo} 2^{-1-}$ spermatocytes. ( $A$ and $B$ ) Representative chromosome spreads from pachytene, early diplotene, and late diplotene $(A)$ wild-type spermatocytes and $(B)$ $S g o 2^{-/-}$spermatocytes. (Scale bars, $5 \mu \mathrm{m}$.) (C) Histogram of SYCP1 localization at centromeres in spreads from wild-type and $\mathrm{SgO}^{-/-}$spermatocytes in early (E), middle (M), and late (L) diplotene. The numbers of SYCP1-positive centromeres scored were $W T$ early, 50/52; $W T$ middle, 46/54; $W T$ late, 88/95; Sgo $2^{-1-}, 72 / 91$; Sgo2 ${ }^{-1-}, 114 / 151$; and Sgo2 ${ }^{-1-}, 100 / 174$. (D) Histogram of the percentage of paired centromeres on chromosomes from wild-type and $\mathrm{Sgo} 2^{-1-}$ spermatocytes. The numbers of paired centromeres were WT early, 48/52; WT middle, 46/54; WT late, 67/98; Sgo2 ${ }^{-1-}, 61 / 91$; Sgo $2^{-1-}, 80 / 151$; and Sgo $2^{-1-}$, 59/174. (E) SYCP1 localization to paired and unpaired centromeres. $(E)$ Paired $(P)$ and unpaired $(U)$ centromeres from all stages of diplotene ( $D$ above) were classified according to their SYCP1 staining. The numbers of centromeres scored were WT paired, 167/167; WT unpaired, 17/ 34; Sgo2 $2^{-1-}$ paired, 200/200; and Sgo $2^{-1-}$ unpaired, 89/216. The significance of differences between samples was evaluated using Fisher's exact test. $* * P<0.01, * * * P<0.001, * * * * P<0.0001$. 


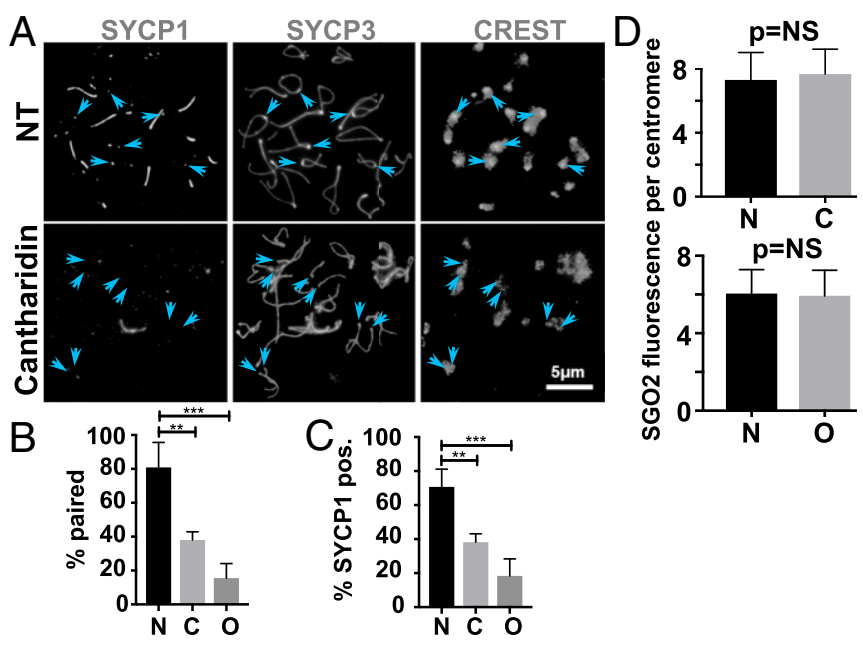

Fig. 4. Phosphatase activity is needed for SYCP1 to persist at centromeres in diplotene. Cultured spermatocytes were treated with okadaic acid or cantharidin. Chromosome spreads were then evaluated using indirect immunofluorescence microscopy. The presence of SYCP1 at centromeres and the fraction of chromosomes in each spread with paired centromeres (stained with CREST antibodies) were scored. $(A)$ Representative images of chromosome spreads that were not treated (NT) or treated with cantharidin. (Scale bar, $5 \mu \mathrm{m}$ for all panels.) Arrowheads indicate examples of paired centromeres (Top) and unpaired centromeres (Bottom). One hundred diplotene chromosome spreads were scored for SCYP1 localization to centromeres and centromere pairing. $(B)$ The percentage of chromosomes in each spread with SYCP1 at the centromeres. Averages and SDs are N (not treated), $80.9 \pm$ $14.7 \%$; C (cantharidin), $38.0 \pm 4.9 \%$; and O (okadaic acid), $15.5 \pm 8.6 \%$. (C) The percentage of chromosomes in each spread with paired centromeres. Averages and SDs are N (not treated), $70.7 \pm 10.4 \%$; C (cantharidin), $38.2 \pm$ $4.9 \%$; and O (okadaic acid), $18.4 \pm 10.0 \%$. One hundred chromosome spreads were scored for each treatment. Significance was evaluated using Student's $t$ test. $(D)$ Histogram showing the relative amount of SGO2 on centromeres of untreated (N) or cantharidin (C)- or okadaic acid (O)-treated spermatocytes. ${ }^{* *} P<0.01, * * * P<0.001$.

Shugoshin Promotes Disjunction of Nonexchange Chromosomes. In budding yeast, it has been possible to demonstrate directly that centromere pairing in prophase is necessary for subsequent disjunction of nonexchange partner chromosomes in anaphase I (60). Since shugoshin is acting at the centromeres in this interval, we tested whether it is important for the meiotic segregation of nonexchange chromosomes. In the mouse model, there is no established system for following the fate of nonexchange chromosomes, so we addressed this question using budding yeast, which has a single shugoshin gene, SGO1. Yeast sgo1s (deletion) mutants show only low levels of meiosis I nondisjunction (of exchange chromosomes), but severe defects in meiosis II (61). As was first shown in Drosophila $(23,24)$, the meiosis II defect is due to a failure to protect centromeric cohesion at the metaphase I to anaphase I transition. We first monitored the requirement for $S G O 1$ for centromere pairing using a pair of centromere-containing plasmids that act as nonexchange minichromosome partners in meiosis (62). These minichromosomes do not experience exchanges, yet they disjoin properly in most meioses $(4,63,64)$. Cells bearing the minichromosomes were induced to enter meiosis, chromosome spreads were prepared, and pachytene spreads (as judged by Zip1 morphology) were scored for the association of the two minichromosomes, which were tagged at their centromeres with GFP and tdTomato (Fig. 5A). The distances between the red and green foci marking the minichromosomes was measured in wild-type (SGO1) cells and cells that do not express SGO1 in meiosis (sgol-md) (65). Foci with center-to-center distances of less than $0.6 \mu \mathrm{m}$ were as scored as "paired" (as in Fig. $5 A, T o p$ ) while those farther apart were scored as "unpaired" (as in Fig. 5A, Bottom).
The sgol-md mutants show considerable centromere pairing in pachytene, although at slightly lower levels than the control (Fig. $5 B)$. Deletion of the $\mathrm{SC}$ gene $Z I P 1$ reduces pairing to a few percent in these assays (62). Thus, as was seen in mice (Fig. 3), shugoshin is not essential for establishing centromere paring. To test whether Sgo1 is necessary for the persistence of centromere pairing after pachytene exit, as it is in spermatocytes (Fig. $3 D$ ), cells were synchronously released from a pachytene arrest and centromere pairing was scored. The arrest/release was achieved by using strains in which the NDT80 meiotic transcription factor was under the control of an estradiol-inducible promotor (43, 66). Following pachytene release cells synchronously pass through diplotene and by $2 \mathrm{~h}$ begin entering early metaphase (SI Appendix, Fig. S4). Centromere pairing of minichromosomes tagged with mTurquoise or mVenus was scored (Fig. $5 C$ and $D$ ). In pachytene cells (just before addition of estradiol) centromeres were paired in most cells in both wild-type and sgol mutants (Fig. 5D). As in the spermatocytes, centromere pairing levels diminished as cells continued meiotic progression (Fig. 5D). By early metaphase the sgol mutants had significantly lower levels of pairing than the wild-type cells (Fig. $5 D$ ). Therefore, as in mouse spermatocytes, centromere pairing is naturally lost following pachytene and shugoshin slows the loss of pairing after pachytene exit.
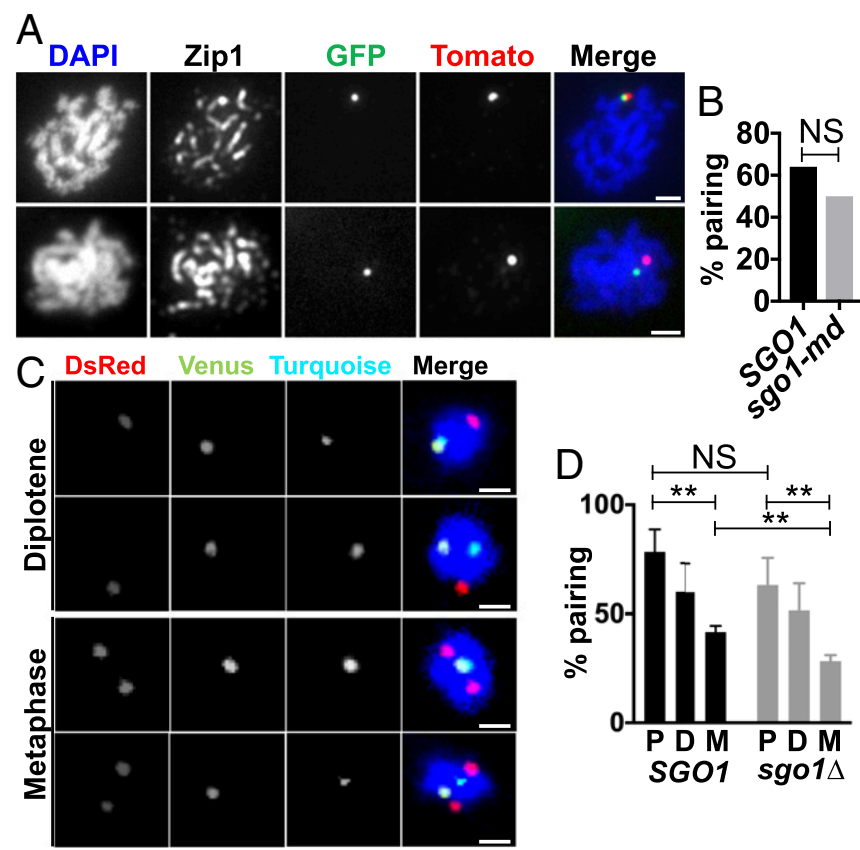

Fig. 5. Shugoshin is not needed for centromere pairing in pachytene but is required for nonexchange segregation. (A) Representative chromosome spreads showing examples of paired (Top) and unpaired (Bottom) minichromosome centromeres (both images from the sgo1-md strain). Chromosome spreads were stained with DAPI to show chromatin, anti-Zip1 antibody to show the SC, and anti-GFP and DsRed to show the locations of the centromere-proximal tags. (Scale bars, $1 \mu \mathrm{m}$.) $(B)$ Histogram showing percentage of centromere pairing in each strain (SGO1, $n=50$ spreads; sgo1-md $n=100$ spreads). (C) Representative micrographs showing examples of paired (Top) and unpaired (Bottom) minichromosome centromeres for both diplotene and metaphase cells (all images from the SGO1 strain DJE90). Cells expressed mVenus-lacl and tetR-mTurquoise which bound to lac operator and tet operator arrays that were adjacent to the centromeres on the two minichromosomes. Spindle pole bodies are shown in red (SPC42-DSRed). Chromosomes were stained with DAPI. (Scale bars, $1 \mu \mathrm{m}$.) (D) Histogram showing percentage of pairing in pachytene, diplotene, and early-metaphase cells ( $P, D$, and $\mathrm{M}$, respectively) in SGO1 and sgo1s cells. Experiments were performed in three replicates of 20 cells each. Statistical comparisons were performed with an unpaired $t$ test. For all histograms, NS, not significant and $* * P<0.01$. 
In both spermatocytes and budding yeast, centromere pairing occurs in prophase and in yeast it is necessary for nonexchange disjunction in anaphase $(14,15)$ - even though the pairing has largely dissolved well before anaphase (Fig. 5D). We have shown the centromere pairing is largely intact in shugoshin mutants but shugoshin affects centromere biology after pachytene exit, as centromere pairing and SC structures at centromeres are lost faster in shugoshin mutants.

To determine whether shugoshin is involved in later events in nonexchange centromere behavior, we examined the segregation of nonexchange minichromosomes in anaphase I (Fig. 6A). Fluorescence microscopy was used to determine whether minichromosomes (marked by tdTomato and GFP foci) segregated to opposite poles in anaphase I cells. In the wild-type control, the minichromosomes nondisjoined in about $26 \%$ of meioses while in the sgo1-md mutants they nondisjoined in $\sim 50 \%$ of meiosesconsistent with random segregation (Fig. 6B). Thus, although most minichromosomes pair at their centromeres in pachytene in sgol mutants (Fig. $5 D$ ), the pairing does not ensure disjunction. We also tested the role of Sgo1 in promoting the disjunction of authentic yeast chromosomes. In these experiments the yeast carried either a normal chromosome $V$ pair or a pair of homeologous chromosome $V \mathrm{~s}$ (one from Saccharomyces cerevisiae and one from Saccharomyces bayanus) that do not experience crossovers in meiosis because of sequence divergence (67). Both chromosome pairs were tagged with GFP at the centromeres.

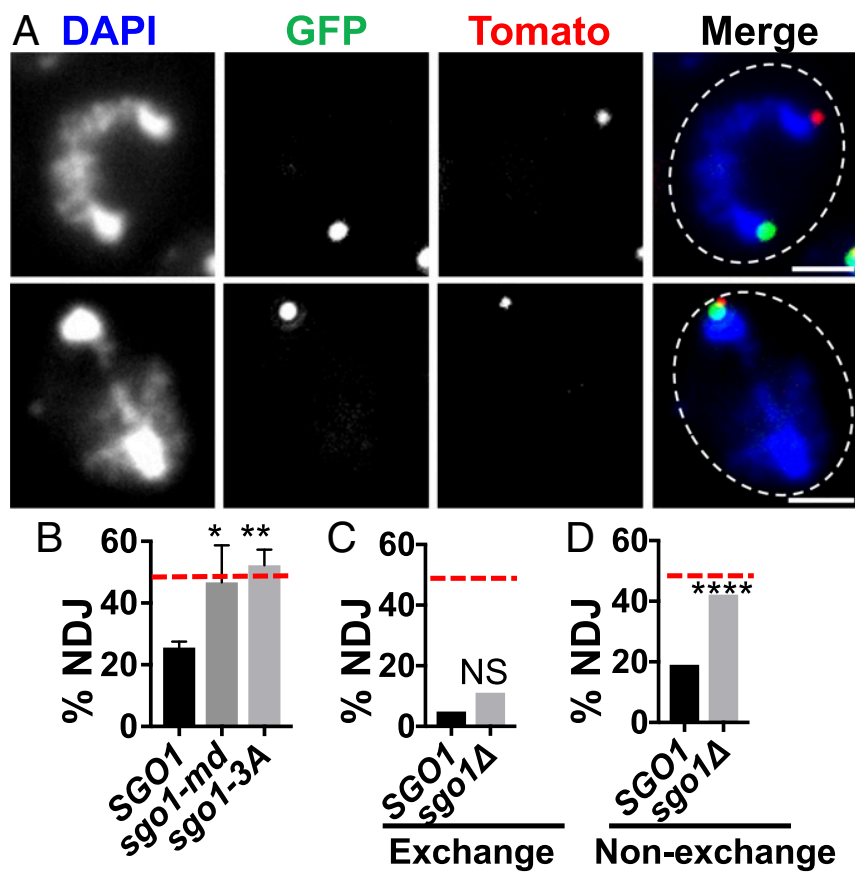

Fig. 6. Shugoshin is required for nonexchange segregation of minichromosomes. (A) Representative anaphase cells showing disjoined (Top) and nondisjoined (Bottom) minichromosomes. Cells were stained with DAPI to show chromatin. Locations of minichromosome centromeres were detected by GFP and tdTomato fluorescence. (Scale bars, $1 \mu \mathrm{m}$.) (B) Histogram showing nondisjunction frequencies of minichromosomes in SGO1, sgo1-md, and sgo1-3A cells (three replicates of 30 cells each were analyzed for all three strains). Statistical comparisons were performed using an unpaired $t$ test. (C) Histogram showing nondisjunction frequencies of homologous chromosomes in SGO1 and sgo1d cells. ( $n=122$ cells for SGO1 and 90 cells for sgo1 $1 \Delta$. (D) Histogram showing nondisjunction frequencies of homeologous chromosomes in SGO1 and sgo1 $1 \Delta$ cells. ( $n=121$ cells for both strains nondisjunction frequencies were $19.0 \%$ vs. $42.1 \%, P<0.0001)$. (B-D) Red dashed line equals the level of nondisjunction expected for random segregation. $(C$ and $D$ ) Statistical comparisons were performed with Fisher's exact test For all histograms, NS, not significant and $* P<0.05$, $* * P<0.01, * * * * P<0.0001$
Deletion of $S G O 1$ (sgols) resulted in a small increase in nondisjunction frequency of the homologous chromosome $V_{\mathrm{s}}$ (Fig. $6 C$ ), consistent with earlier studies (61). In contrast, the nonexchange, homeologous pair exhibited a significant increase in nondisjunction when $S G O 1$ was deleted, consistent with nearly random segregation (Fig. $6 D$ ).

The PP2A inhibitor experiments suggested that Sgo2 (in mice) acts to protect centromere pairing through recruitment of PP2A. Is Sgo1 of yeast promoting nonexchange disjunction by recruiting PP2A? To test this, we took advantage of the sgol-3A allele in which three critical contact amino acids required for $\mathrm{Sgo} 1$ to recruit PP2A to centromeres are converted to alanines (29). This mutant, which exhibits normal loading of Sgo1 to meiotic kinetochores (29), showed random segregation of the nonexchange partners, providing strong support for the model that Sgo1 promotes nonexchange segregation through the recruitment of PP2A to centromeres.

These experiments show that shugoshin is not needed for the establishment of centromere pairing, that centromere pairing dissolves after pachytene exit, and that shugoshin slows the dissolution of centromere pairing. In both nematodes and rice, SCs disassemble prematurely in the absence of shugoshin $(30,34)$. Thus, it could be that the protection of SC components at centromeres, observed in our studies, reflects a more general conserved role for shugoshins in modulating SC stability and the timing of its disassembly.

While previous work demonstrated that prophase centromere pairing is essential for nonexchange disjunction in yeast $(14,15)$, the results presented here reveal that centromere pairing is not sufficient to ensure disjunction-since in yeast SGO1 mutants most centromeres are paired upon pachytene exit. Earlier studies found that in wild-type cells, centromeric SC proteins disappear before chromosomes begin to orient on the spindle in early metaphase (14-17). This observation, coupled with our findings, suggests that centromere pairing might be a precondition for setting up the mechanism that later promotes biorientation in anaphase. Centromere pairing cannot be directly mediating biorientation since SC proteins are normally gone from the centromeres, and centromere pairing is dissolved, before biorientation is accomplished. The observation that nonexchange partners in Drosophila appear to be tethered by threads of pericentromeric heterochromatin during the metaphase biorientation process (68) suggests the model that prophase centromere pairing could provide a platform for the establishment of centromeric connections between nonexchange partners. The fact that nonexchange segregation is randomized in yeast shugoshin mutants raises the possibility that shugoshin is not essential for centromere pairing, per se, but instead for the formation or maintenance of a structure or process that promotes biorientation. By this model, the low levels of meiosis I nondisjunction of native chromosomes in shugoshin mutants may reflect the times at which these chromosomes fail to experience exchanges and rely upon a centromere pairing-based mechanism to ensure their disjunction in meiosis I.

\section{Materials and Methods}

The mouse and yeast strains, reagents, and methods for this project are described in SI Appendix. The Oklahoma Medical Research Foundation Animal Care and Use Committee approved all animal protocols. For mouse cytology experiments, staging of chromosome spreads in diplotene was based on the extent of SYCP1 staining. Established approaches were employed for visualizing chromosomes in surface spreads $(16,50,69,70)$.

ACKNOWLEDGMENTS. The authors thank the members of the Program in Cell Cycle and Cancer Biology for their constructive comments during this project. A.M.P. is funded by Award BFU2017-89408-R from the Spanish Ministry of Economy and Competitiveness and his program is supported by the Programa de Apoyo a Planes Estratégicos de Investigación de Estructuras de Investigación de Excelencia, which is cofunded by the Castilla-León autonomous government and the European Regional Development Fund (Award CLC-2017-01). R.J.P. was supported by Centers of Biomedical Research Excellence Grant GM103636 and March of Dimes Grant FY14-256. D.S.D. was supported by NIH Grant R01 GM087377. 
1. Hunter N (2015) Meiotic recombination: The essence of heredity. Cold Spring Harb Perspect Biol 7:a016618.

2. Kurdzo EL, Dawson DS (2015) Centromere pairing-Tethering partner chromosomes in meiosis I. FEBS J 282:2458-2470.

3. Carpenter AT (1973) A meiotic mutant defective in distributive disjunction in Drosophila melanogaster. Genetics 73:393-428.

4. Dawson DS, Murray AW, Szostak JW (1986) An alternative pathway for meiotic chromosome segregation in yeast. Science 234:713-717.

5. Guacci V, Kaback DB (1991) Distributive disjunction of authentic chromosomes in Saccharomyces cerevisiae. Genetics 127:475-488.

6. Hawley RS, et al. (1992) There are two mechanisms of achiasmate segregation in Drosophila females, one of which requires heterochromatic homology. Dev Genet 13 440-467.

7. Woods LM, et al. (1999) Chromosomal influence on meiotic spindle assembly: Abnormal meiosis I in female Mlh1 mutant mice. J Cell Biol 145:1395-1406.

8. Cheng EY, et al. (2009) Meiotic recombination in human oocytes. PLoS Genet 5: e1000661.

9. Oliver TR, et al. (2008) New insights into human nondisjunction of chromosome 21 in oocytes. PLoS Genet 4:e1000033.

10. Fledel-Alon A, et al. (2009) Broad-scale recombination patterns underlying proper disjunction in humans. PLoS Genet 5:e1000658.

11. Tease C, Hartshorne GM, Hultén MA (2002) Patterns of meiotic recombination in human fetal oocytes. Am J Hum Genet 70:1469-1479.

12. Dernburg AF, Sedat JW, Hawley RS (1996) Direct evidence of a role for heterochromatin in meiotic chromosome segregation. Cell 86:135-146.

13. Ding DQ, Yamamoto A, Haraguchi T, Hiraoka Y (2004) Dynamics of homologous chromosome pairing during meiotic prophase in fission yeast. Dev Cell 6:329-341.

14. Gladstone MN, Obeso D, Chuong H, Dawson DS (2009) The synaptonemal complex protein Zip1 promotes bi-orientation of centromeres at meiosis I. PLoS Genet 5 e1000771.

15. Newnham L, Jordan P, Rockmill B, Roeder GS, Hoffmann E (2010) The synaptonema complex protein, Zip1, promotes the segregation of nonexchange chromosomes at meiosis I. Proc Natl Acad Sci USA 107:781-785.

16. Bisig CG, et al. (2012) Synaptonemal complex components persist at centromeres and are required for homologous centromere pairing in mouse spermatocytes. PLoS Genet 8:e1002701.

17. Qiao H, et al. (2012) Interplay between synaptonemal complex, homologous recombination, and centromeres during mammalian meiosis. PLoS Genet 8:e1002790.

18. Takeo S, Lake CM, Morais-de-Sá E, Sunkel CE, Hawley RS (2011) Synaptonema complex-dependent centromeric clustering and the initiation of synapsis in Drosophila oocytes. Curr Biol 21:1845-1851.

19. Unhavaithaya Y, Orr-Weaver TL (2013) Centromere proteins CENP-C and CAL1 functionally interact in meiosis for centromere clustering, pairing, and chromosome segregation. Proc Natl Acad Sci USA 110:19878-19883.

20. Khetani RS, Bickel SE (2007) Regulation of meiotic cohesion and chromosome core morphogenesis during pachytene in Drosophila oocytes. I Cell Sci 120:3123-3137.

21. Tanneti NS, Landy K, Joyce EF, McKim KS (2011) A pathway for synapsis initiation during zygotene in Drosophila oocytes. Curr Biol 21:1852-1857.

22. Rankin S (2015) Complex elaboration: Making sense of meiotic cohesin dynamics. FEBS J 282:2426-2443.

23. Kerrebrock AW, Miyazaki WY, Birnby D, Orr-Weaver TL (1992) The Drosophila meiS332 gene promotes sister-chromatid cohesion in meiosis following kinetochore dif ferentiation. Genetics 130:827-841.

24. Kerrebrock AW, Moore DP, Wu JS, Orr-Weaver TL (1995) Mei-S332, a Drosophila protein required for sister-chromatid cohesion, can localize to meiotic centromere regions. Cell 83:247-256.

25. Kitajima TS, et al. (2006) Shugoshin collaborates with protein phosphatase $2 \mathrm{~A}$ to protect cohesin. Nature 441:46-52.

26. Lee J, et al. (2008) Unified mode of centromeric protection by shugoshin in mam malian oocytes and somatic cells. Nat Cell Biol 10:42-52.

27. Rattani A, et al. (2013) Sgol2 provides a regulatory platform that coordinates essentia cell cycle processes during meiosis I in oocytes. eLife 2:e01133.

28. Riedel CG, et al. (2006) Protein phosphatase 2A protects centromeric sister chromatid cohesion during meiosis I. Nature 441:53-61.

29. Xu Z, et al. (2009) Structure and function of the PP2A-shugoshin interaction. Mol Cell 35:426-441.

30. Bohr T, Nelson CR, Giacopazzi S, Lamelza P, Bhalla N (2018) Shugoshin is essential for meiotic prophase checkpoints in C. elegans. Curr Biol 28:3199-3211.e3.

31. Hara K, et al. (2014) Structure of cohesin subcomplex pinpoints direct shugoshin-Wap antagonism in centromeric cohesion. Nat Struct Mol Biol 21:864-870.

32. Orth M, et al. (2011) Shugoshin is a Mad1/Cdc20-like interactor of Mad2. EMBO J 30: 2868-2880.

33. Tanno Y, et al. (2010) Phosphorylation of mammalian Sgo2 by Aurora B recruits PP2A and MCAK to centromeres. Genes Dev 24:2169-2179.

34. Wang M, et al. (2011) OsSGO1 maintains synaptonemal complex stabilization in addition to protecting centromeric cohesion during rice meiosis. Plant J 67:583-594.

35. Ishiguro T, Tanaka K, Sakuno T, Watanabe Y (2010) Shugoshin-PP2A counteracts casein-kinase-1-dependent cleavage of Rec8 by separase. Nat Cell Biol 12:500-506.

36. Katis VL, et al. (2010) Rec8 phosphorylation by casein kinase 1 and Cdc7-Dbf4 kinase regulates cohesin cleavage by separase during meiosis. Dev Cell 18:397-409.

37. Rumpf C, et al. (2010) Casein kinase 1 is required for efficient removal of Rec 8 during meiosis I. Cell Cycle 9:2657-2662.
38. Cahoon CK, Hawley RS (2016) Regulating the construction and demolition of the synaptonemal complex. Nat Struct Mol Biol 23:369-377.

39. Jordan PW, Karppinen J, Handel MA (2012) Polo-like kinase is required for synaptonemal complex disassembly and phosphorylation in mouse spermatocytes. J Cell Sci 125:5061-5072.

40. Tarsounas M, Pearlman RE, Moens PB (1999) Meiotic activation of rat pachytene spermatocytes with okadaic acid: The behaviour of synaptonemal complex components SYN1/SCP1 and COR1/SCP3. J Cell Sci 112:423-434.

41. Sourirajan A, Lichten M (2008) Polo-like kinase Cdc5 drives exit from pachytene during budding yeast meiosis. Genes Dev 22:2627-2632.

42. Argunhan B, et al. (2017) Fundamental cell cycle kinases collaborate to ensure timely destruction of the synaptonemal complex during meiosis. EMBO J 36:2488-2509.

43. Carlile TM, Amon A (2008) Meiosis I is established through division-specific trans lational control of a cyclin. Cell 133:280-291.

44. Matos J, et al. (2008) Dbf4-dependent CDC7 kinase links DNA replication to the segregation of homologous chromosomes in meiosis I. Cell 135:662-678.

45. Sun F, Handel MA (2008) Regulation of the meiotic prophase I to metaphase I transition in mouse spermatocytes. Chromosoma 117:471-485.

46. Anderson LK, Reeves A, Webb LM, Ashley T (1999) Distribution of crossing over on mouse synaptonemal complexes using immunofluorescent localization of MLH protein. Genetics 151:1569-1579.

47. Jagiello G, Fang JS (1979) Analyses of diplotene chiasma frequencies in mouse oocytes and spermatocytes in relation to ageing and sexual dimorphism. Cytogenet Cell Genet 23:53-60.

48. Speed RM (1977) The effects of ageing on the meiotic chromosomes of male and female mice. Chromosoma 64:241-254.

49. Zelazowski MJ, et al. (2017) Age-dependent alterations in meiotic recombination cause chromosome segregation errors in spermatocytes. Cell 171:601-614.e13.

50. Guiraldelli MF, Eyster C, Wilkerson JL, Dresser ME, Pezza RJ (2013) Mouse HFM1/Mer3 is required for crossover formation and complete synapsis of homologous chromosomes during meiosis. PLoS Genet 9:e1003383.

51. Lipkin SM, et al. (2002) Meiotic arrest and aneuploidy in MLH3-deficient mice. Nat Genet 31:385-390.

52. Holloway JK, Booth J, Edelmann W, McGowan CH, Cohen PE (2008) MUS81 generates a subset of MLH1-MLH3-independent crossovers in mammalian meiosis. PLoS Genet 4: e1000186.

53. Gómez R, et al. (2007) Mammalian SGO2 appears at the inner centromere domain and redistributes depending on tension across centromeres during meiosis II and mitosis. EMBO Rep 8:173-180.

54. Marston AL (2015) Shugoshins: Tension-sensitive pericentromeric adaptors safe guarding chromosome segregation. Mol Cell Biol 35:634-648.

55. Llano E, et al. (2008) Shugoshin-2 is essential for the completion of meiosis but not for mitotic cell division in mice. Genes Dev 22:2400-2413.

56. La Salle S, Sun F, Handel MA (2009) Isolation and short-term culture of mouse spermatocytes for analysis of meiosis. Methods Mol Biol 558:279-297.

57. Honkanen RE (1993) Cantharidin, another natural toxin that inhibits the activity of serine/threonine protein phosphatases types 1 and 2A. FEBS Lett 330:283-286.

58. Bialojan C, Takai A (1988) Inhibitory effect of a marine-sponge toxin, okadaic acid, on protein phosphatases. Specificity and kinetics. Biochem J 256:283-290.

59. Pinto BS, Orr-Weaver TL (2017) Drosophila protein phosphatases 2A B' Wdb and Wrd regulate meiotic centromere localization and function of the MEI-S332 Shugoshin. Proc Natl Acad Sci USA 114:12988-12993.

60. Kurdzo EL, Chuong HH, Evatt JM, Dawson DS (2018) A ZIP1 separation-of-function allele reveals that centromere pairing drives meiotic segregation of achiasmate chromosomes in budding yeast. PLOS Genet 14:e1007513.

61. Marston AL, Tham WH, Shah $\mathrm{H}$, Amon A (2004) A genome-wide screen identifies genes required for centromeric cohesion. Science 303:1367-1370.

62. Kurdzo EL, Obeso D, Chuong H, Dawson DS (2017) Meiotic centromere coupling and pairing function by two separate mechanisms in Saccharomyces cerevisiae. Genetics 205:657-671.

63. Mann C, Davis RW (1986) Meiotic disjunction of circular minichromosomes in yeast does not require DNA homology. Proc Natl Acad Sci USA 83:6017-6019.

64. Sears DD, Hegemann JH, Hieter P (1992) Meiotic recombination and segregation of human-derived artificial chromosomes in Saccharomyces cerevisiae. Proc Natl Acad Sc USA 89:5296-5300.

65. Monje-Casas F, Prabhu VR, Lee BH, Boselli M, Amon A (2007) Kinetochore orientation during meiosis is controlled by Aurora $B$ and the monopolin complex. Cell 128 477-490.

66. Benjamin KR, Zhang C, Shokat KM, Herskowitz I (2003) Control of landmark events in meiosis by the CDK Cdc28 and the meiosis-specific kinase Ime2. Genes Dev 17 1524-1539.

67. Maxfield Boumil R, Kemp B, Angelichio M, Nilsson-Tillgren T, Dawson DS (2003) Meiotic segregation of a homeologous chromosome pair. Mol Genet Genomics 268: $750-760$.

68. Hughes SE, et al. (2009) Heterochromatic threads connect oscillating chromosomes during prometaphase I in Drosophila oocytes. PLoS Genet 5:e1000348.

69. Kemp B, Boumil RM, Stewart MN, Dawson DS (2004) A role for centromere pairing in meiotic chromosome segregation. Genes Dev 18:1946-1951.

70. Handel MA, Caldwell KA, Wiltshire T (1995) Culture of pachytene spermatocytes for analysis of meiosis. Dev Genet 16:128-139. 